\title{
Amlodipine Induced Gingival Overgrowth and its Nonsurgical Management-A Case Report
}

\author{
Authors \\ Dr Suchetha Aghanashini, M.D.S ${ }^{1}$, Dr Suma Prashanth ${ }^{2}$, Dr Apoorva. S.M, M.D.S \\ Dr Darshan. B.M, M.D.S ${ }^{4}$, Dr Sapna.N, M.D.S ${ }^{5}$, Dr Divya Bhat, M.D.S \\ ${ }^{1}$ Professor and Head of the Department, Department of Periodontics \\ ${ }^{2}$ Post Graduate Student, Department of Periodontics \\ ${ }^{3,4,5}$ Reader, Department of Periodontics \\ ${ }^{6}$ Lecturer, Department of Periodontics \\ D.A.P.M. R V Dental College Bangalore, India
}

\begin{abstract}
Gingival enlargement or overgrowth is a well-known adverse effect associated with three major groups of drugs i.e., anticonvulsants, calcium channel blockers, and immunosuppressants. Among calcium channel blockers, nifedipine is the commonest cause for drug induced gingival overgrowth with the incidence of $10 \%$ whereas case report of gingival enlargement due to amlodipine are very less compared to nifedipine. Hence, we hereby report a case of amlodipine induced gingival overgrowth which intends to highlight the efficacy of conservative management with scaling, root planing and drug substitution.

Keywords: gingival overgrowth, amlodipine, scaling and root planing, drug substitution.
\end{abstract}

\section{Introduction}

Gingival enlargement or gingival overgrowth is characterized by an increase in the size of gingiva. Gingival enlargements can be classified depending on the etiologic factors and pathologic changes, according to location and distribution and/or according to the degree of enlargement.

According to etiopathogenesis, enlargements could be inflammatory, drug influenced, those associated with systemic conditions or diseases, neoplastic or false enlargements. Based on location, enlargements could be marginal, papillary or diffuse. According to distribution they can be localized or generalized. Localized enlargements could be further divided into three sub-types, "isolated, discrete" or "regional". Enlargements which are "isolated" are those confined to gingiva adjacent to single or two teeth (e.g., gingival/periodontal abscess). Lesions which are "discrete" include isolated sessile or pedunculated, tumor-like enlargements (e.g., fibroma/pyogenic granuloma). Enlargements which are "regional" are those with the involvement of gingiva around three or more teeth in one or multiple areas of the mouth (e.g., inflammatory enlargement associated with mouth breathing in maxillary and mandibular anterior region). Enlargement which are "generalized" means involvement of gingiva adjacent to almost all the teeth present (e.g., drug influenced gingival overgrowth). ${ }^{1}$

Drug-induced gingival enlargement presents as abnormal growth of the gingiva due to an adverse 
drug reaction in patients treated with anticonvulsants, immunosuppressants, and calcium channel blockers.

As gingival enlargement develops, it affects the routine oral hygiene practice and may interfere with masticatory functions. It gradually becomes a source of pain because of the inflammation and the condition often leads to disfigurement. There appears to be variability in the extent and severity of the gingival changes. ${ }^{2}$

\section{Drugs Causing Gingival Enlargement}

Drugs causing gingival enlargement include calcium channel blockers like Amlodipine, Diltiazem, Felodipine, Isradipine, Nicardipine, Nifedipine, Nisoldipine, Verapamil ${ }^{3}$, Manidipine ${ }^{4}$, immunosuppressants like tacrolimus and cyclosporine ${ }^{5}$, anticonvulsants like phenobarbitone, primidone, and valproic acid, miscellaneous like erythromycin and sertraline, etc. $^{6}$

\section{Clinical Manifestations of Gingival Enlargement}

Frequently appear within one to three months, after the initiation of treatment with the associated medications. Gingival overgrowth begins at the interdental papillae and is more frequently found in the anterior segment of the labial surfaces. Gradually, gingival lobulations are formed that may appear inflamed or fibrotic depending on the degree of local factor-induced inflammation. However, the fibrotic enlargement is confined to the attached gingiva but may extend coronally causing the extensive disfigurement of gingiva. ${ }^{7}$

Several factors namely; age, genetic predisposition, presence of pre-existing plaque, and gingival inflammation influence the relationship between the drugs and gingival tissue. There is a variable gingival response in patients taking drugs and can be categorized into "responders" and "non-responders". Furthermore, within the group of patients those who develop this unwanted effect, there appears to be variability in the extent and severity of the gingival changes. ${ }^{2}$

Despite the similar conditions concerning plaque and amlodipine dosage, only some of the drug receivers become affected with hyperplasia and others do not, probably this can be mainly because of the biological differences among human beings, such as the existence of different subgroups of gingival fibroblasts.

\section{Amlodipine Induced Enlargement}

A newer agent of dihydropyridine is amlodipine which is used for the treatment of hypertension and angina, was first reported for causing gingival overgrowth as a side effect by Seymour et al in 1994. The other members of dihydropyridine include nifedipine, nicardipine, isoradipine, nitrendipine, and felodipine. These drugs cause coronary and peripheral arterial vasodilatation at a dose of 2.5 or 5 grams (alone or in combination with atenolol), with the following adverse effects of headaches, facial flushing, dizziness, edema, gingival hyperplasia, and the drug is detectable in gingival crevicular fluid. ${ }^{8}$

The mechanism through which calcium channel blockers causes gingival enlargement is by inhibiting the intracellular $\mathrm{Ca}^{2+}$ uptake thereby stimulating gingival fibroblasts. Human gingival fibroblasts produce collagenous protein when the cells are exposed simultaneously to calcium channel blockers and elevated levels of interleukin$1 \beta$ (a proinflammatory cytokine) in inflamed gingival tissues. Fibroblasts are targeted by interleukin-6 which trigger the proliferation of fibroblasts and exert the positive regulation on collagen and glycosaminoglycans synthesis. Hence this cytokine has been proposed to play a pathogenic role in fibrotic gingival enlargement. ${ }^{9}$ The prevalence of amlodipine-induced gingival hypertrophy has been shown to be between $1.7 \%$ and $3.3 \%{ }^{8}$ Amlodipine-induced gingival enlargement is comparatively less prevalent than other calcium channel blocker. ${ }^{10}$ The highest prevalence of gingival enlargement was in the fourth decade of life, with the incidence of overgrowth being higher in males compared to females. ${ }^{11}$

The management for amlodipine induced gingival enlargement can be started with the nonsurgical approach of scaling and root planing. Patient's 
physician has to be consulted regarding drug substitution or withdrawal of the drug. After 3 to 6 months of drug substitution, surgical excision of retained gingival overgrowth will be planned using the techniques of gingivectomy or gingivoplasty to restore the normal shape and contour of the gingiva. Patient will be instructed to maintain good oral hygiene.

Amlodipine can be substituted with verapamil, diltiazem, angiotensin receptor blockers like losartan etc. There was no recurrence of gingival overgrowth observed after amlodipine being substituted for any of the drug mentioned earlier which was reported by AK Srivastava et al. ${ }^{10}$

This report presents a case of amlodipine-induced gingival overgrowth where it was treated in the following phases: (1) thorough Phase-1 therapy, (2) substitution of the drug, (3) surgical excision of tissue for biopsy and (4) maintenance and supportive therapy.

\section{Case Report}
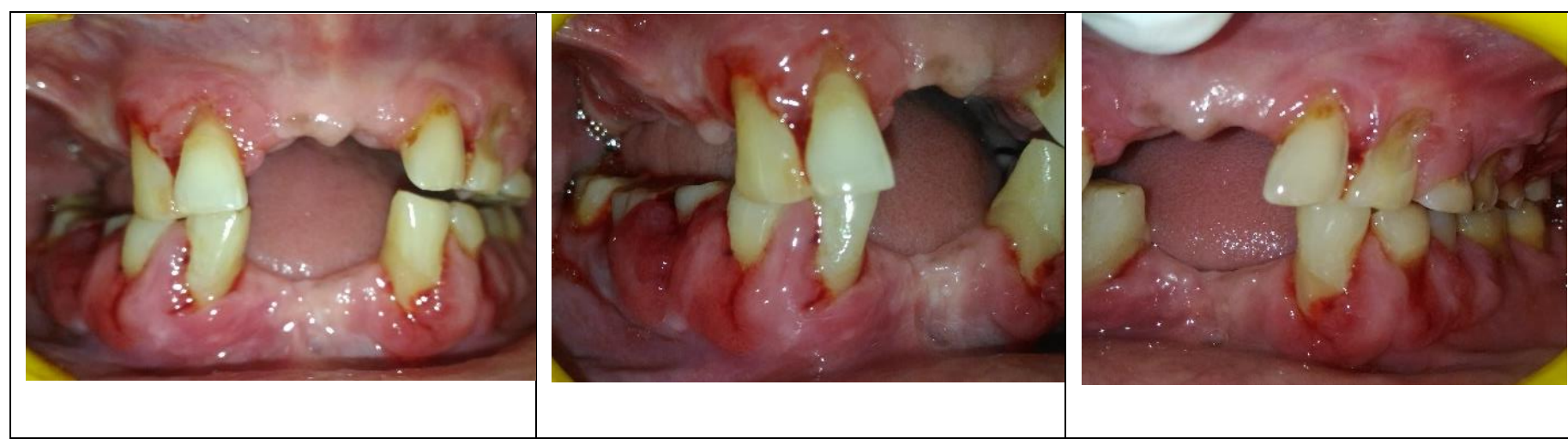

Preoperative view showing gingival overgrowth (frontal and lateral view)

\section{Treatment}

Treatment for drug-induced gingival overgrowth comprises of non-surgical and/or surgical therapies. Non-surgical treatment, Phase I therapy was done which included the scaling and root planing for removing the local factors and diseased cementum, wherever exposed. Instructions were given to the patient to maintain good oral hygiene. After completion of Phase I therapy, patient's maintenance phase was observed for about 4 weeks. In the subsequent follow-up, clinical
A 62-year-old female patient reported to Department of Periodontics, DAPMRV Dental College Bengaluru, with the chief complaint of gingival enlargement and wanted to replace her missing front teeth. On taking a detailed case history, the patient reported that she got her front teeth extracted 3 months back due to severe mobility. Medical and drug history of the patient revealed that patient was known hypertensive and was on medication for last 10 years (Amlodipine $5 \mathrm{mg}$ once daily).

On intraoral examination, Gingiva was pink in color with reddish discoloration wrt 12, 13, 22-28, 33-37, 43-47. Marginal gingiva was rolled out with loss of normal gingival scalloping. On palpation, gingiva was soft and oedematous in consistency. Diffuse gingival enlargement was appreciated, and the surface was lobulated almost covering coronal third of 24,44,45,46. The probing elicited generalized bleeding. Based on the patient's history and clinical features, a clinical diagnosis of amlodipine-induced gingival overgrowth (AIGO) was made. examination of the overall gingival tissue showed reduction of the inflammatory component, bleeding on probing, the erythema, edema and surface shininess. Incisional biopsy was done for histopathological confirmation, tissue was excised from the region of 46 .

\section{Biopsy}

The histopathological report revealed stratified squamous parakeratinized epithelium covering a fibrocellular tissue. The underlying connective tissue showed abundant collagen fibers admixed 
with spindle-shaped fibroblast. Dispersion of numerous inflammatory cells throughout the connective tissue was observed.

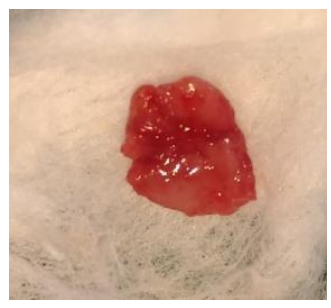

\section{Biopsy specimen}

The patient was referred to a physician for his consent and for substitution of drug (amlodipine) with another antihypertensive drug, for which the physician agreed and substituted amlodipine with losartan $50 \mathrm{mg}$ once daily (angiotensin receptor blocker).

After substitution of the drug, the patient was under maintenance therapy for the next two months. The patient was observed weekly once for a period of next two months. On examination after two months, the overgrowth had subsided almost completely except for minor fibrous nodules remaining in mandibular and maxillary canine region. The patient was referred to Department of Prosthodontics for the replacement of missing teeth.

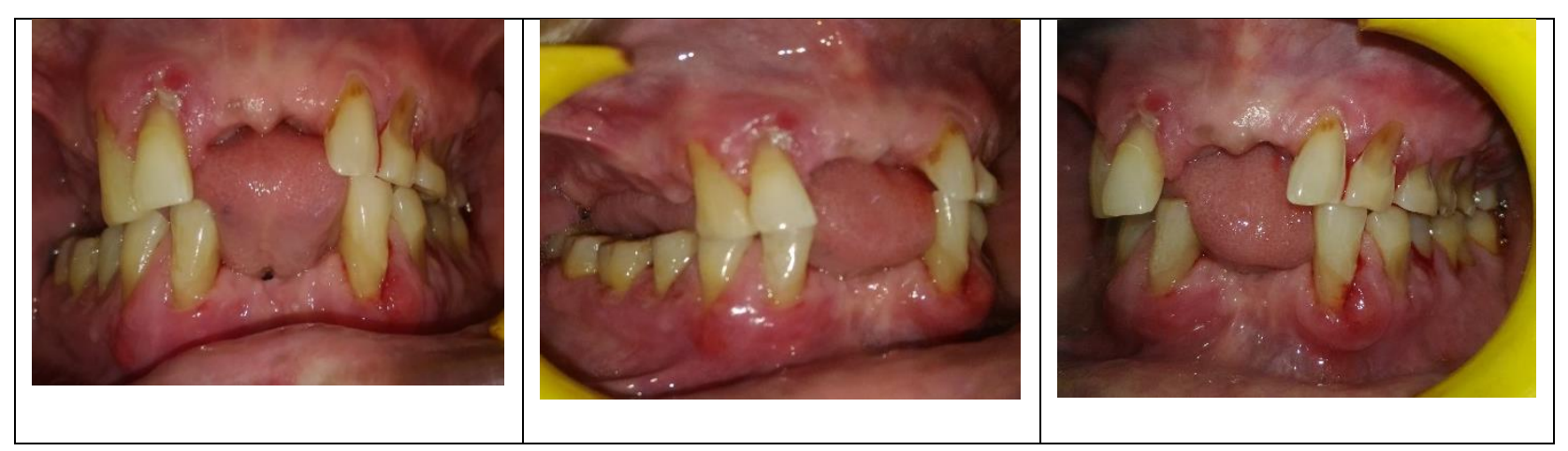

Postoperative view showing regression of gingival overgrowth (frontal and lateral view)

\section{Discussion}

Poor oral hygiene is an important risk factor for drug-induced gingival overgrowth. But there is no clear evidence that bacterial plaque is a contributory factor for the gingival changes. However, two main inflammatory and noninflammatory pathways have been suggested.

The non-inflammatory mechanism develops because of defective collagenase activity due to decreased uptake of folic acid, blockage of aldosterone synthesis, in adrenal cortex and consequent feedback increase in the level of adrenocorticotropic hormone and upregulation of keratinocyte growth factor.

Alternatively, inflammation may develop because of the direct toxic effects of the concentrated drug in crevicular gingival fluid and/or bacterial plaque. This inflammation could lead to the upregulation of several cytokine factors such as transforming growth factor-beta 1 [TGF- $\beta 1]{ }^{11}$
Treatment, in general, focuses on the substitution of drug and effective control of local inflammatory factors such as plaque and calculus. When nonsurgical approach fails to resolve the gingival enlargement, surgical intervention is recommended. Surgery is normally preferred for aesthetic needs before any functional consequences are present. $^{12}$

There are several modalities in treating gingival overgrowth like scalpel, laser, and electrocautery etc.

Sachin Funde et al conducted a study to Compare between Laser, Electrocautery and Scalpel in the treatment of Drug-Induced Gingival Overgrowth in the year 2015 where he reported the advantages and disadvantages of each technique.

It was observed that scalpel has many advantages like ease of use, precise incision with well-defined margins, relatively fast and uneventful healing. It also has many disadvantages like need of anesthesia, excessive bleeding, inadequate 
visibility caused by blood in the operating field, non-sterilized incision cut.

The following advantages of electrocautery were appreciated, where the electrode cuts on its side as well as on its tip, angulated electrode meets the clinical need, cuts are made with ease when the device is set correctly, immediate and consistent hemostasis, the wound was less painful, and the tip is self-disinfecting. Disadvantages of electrocautery includes the requirement of anesthetic agent for cutting, unavoidable burningflesh odor, low tactile sense, does not allow for their use around implants, bone can be damaged, hazardous in an explosive environment, contraindicated in patients with pacemakers, poor postoperative healing who have undergone irradiation, diabetes or blood dyscrasias.

Laser had many advantageous like requirement of minimal or no anesthesia, no harm to dental hard tissues. Their sensible use does not injure the dental pulp, because of low or no heat production and can be used around dental implants. Diode lasers have several advantages when compared to scalpel surgeries. They are portable and compact in design with efficient and reliable benefits for use in soft tissue oral surgical procedures. Laser surgeries are easy to perform with less discomfort, minimal or no bleeding due to sealing of capillaries by protein denaturation and stimulation of clotting factor VII production, shorter healing time with reduced postoperative bleeding and oedema.

On the other hand, disadvantages of laser include eye damage which demands protective glasses. Laser cutting is slower than that with electrosurgery with burning flesh odor. Working area should be free of combustible gases. During laser use, laser plume requires use of a highfiltration face mask. ${ }^{13}$

Apart from surgical intervention few studies have been reported by Chi-Ching Chan in 2018 using noninvasive protocol for managing conditions like Drug-induced gingival enlargement. It is a common condition which can be observed in patients taking immunosuppressive medications following organ transplant surgery. The disfiguring excessive tissue often hampers proper oral hygiene practices, therefore accompanied by periodontitis, tooth mobility, and even pathological tooth migration in extreme cases. A fifty-one-year-old woman had experienced renal transplant surgery 16 years prior to her dental visit and had been taking immunosuppressive drugs including cyclosporine ever since. Following clinical and radiographic examinations, the patient was diagnosed with drug-induced gingival enlargement, pathological tooth migration, severe periodontitis, and missing teeth. Through careful and meticulous nonsurgical debridement, oral hygiene instruction, tooth extraction, and occlusal adjustment, the patient's periodontium was restored to a healthy state without surgical intervention. Moreover, the patient's chewing function was restored by means of removable partial dentures. ${ }^{14}$

In this present case, marked reduction in inflammation and gingival overgrowth was observed after phase-1 therapy and substitution of amlodipine to another drug. Meticulous oral hygiene maintenance by the patient may also be responsible for the reduction in gingival overgrowth.

\section{Conclusion}

Patient had come with a primary complaint of replacing her missing teeth and since she had gingival overgrowth which was drug induced, it was managed conservatively with scaling, root planing and drug substitution. Gingival overgrowth was subsided following which she got her missing teeth replaced with removable prosthesis in department of prosthodontics and patient was satisfied with treatment outcome.

Hence, we can conclude that the conservative approach with scaling and root planing and drug substitution (after physician consent) would be sufficient to reduce the gingival overgrowth induced by chronic medication with amlodipine, rather than more extensive surgical procedures like gingivectomy or gingivoplasty. However, more 
clinical studies are required to prove the effectiveness of the conservative approach.

\section{References}

1 Agrawal AA. Gingival enlargements: Differential diagnosis and review of literature. World Journal of Clinical Cases: WJCC. 2015 Sep 16;3(9):779.

2 Bharti V, Bansal C. Drug-induced gingival overgrowth: the nemesis of gingiva unravelled. Journal of Indian Society of Periodontology. 2013 Mar;17(2):182.

3 Livada R, Shiloah J. Calcium channel blockerinduced gingival enlargement. Journal of human hypertension. 2014 Jan;28(1):10.

4 Thompson AL, Herman WW, Konzelman J, Collins MA. Treating patients with druginduced gingival overgrowth. American Dental Hygienists' Association. 2004 Oct 1;78(4):12

5 Hassan F, Tawfig N, Gobara B. Gingival overgrowth in subjects under immunosuppressive regimens based on tacrolimus or combination of tacrolimus and amlodipine. Dentistry. 2015;5(9):1-4.

6 Patsalos PN, Perucca E. Clinically important drug interactions in epilepsy: interactions between antiepileptic drugs and other drugs. The Lancet Neurology. 2003 Aug 1;2(8):47381.

7 Seymour RA, Ellis JS, Thomason JM, Monkman S, Idle JR. Amlodipine-induced gingival overgrowth. Journal of clinical periodontology. 1994 Apr;21(4):281-3.
8 Madi M, Shetty SR, Babu SG, Achalli S. Amlodipine-induced gingival hyperplasia-a case report and review. The West Indian medical journal. 2015 Jun;64(3):279.

9 Joshi S, Bansal S. A rare case report of amlodipine-induced gingival enlargement and review of its pathogenesis. Case reports in dentistry. 2013;2013.

10 Srivastava AK, Kundu D, Bandyopadhyay P, Pal AK. Management of amlodipine-induced gingival enlargement: Series of three cases. Journal of Indian Society of Periodontology. 2010 Oct;14(4):279.

11 Tejnani A, Mani A, Sodhi NK, Mehta A, Gourkhede S, Thorat V, Marawar P. Incidence of amlodipine-induced gingival overgrowth in the rural population of Loni. Journal of Indian Society of Periodontology. 2014 Mar;18(2):226.

12 Greenstein G. Nonsurgical periodontal therapy in 2000: a literature review. The Journal of the American Dental Association. 2000 Nov 1;131(11):1580-92.

13 Funde S, Baburaj MD, Pimpale SK. Comparison between laser, electrocautery and scalpel in the treatment of drug-induced gingival overgrowth-a case report. IJSS Case Reports and Reviews. 2015 Mar;1(10):27-30.

14 Chang CC, Lin TM, Chan CP, Pan WL. Nonsurgical periodontal treatment and prosthetic rehabilitation of a renal transplant patient with gingival enlargement: a case report with 2-year follow-up. BMC oral health. 2018 Dec;18(1):140. 